\title{
Force-FAK Signaling Coupling at Individual Focal Adhesions Coordinates Mechanosensing and Microtissue Repair
}

\section{Dennis Zhou}

Georgia Institute of Technology https://orcid.org/0000-0001-7448-5395

Marc Fernández-Yagüe

Georgia Institute of Technology

Elijah Holland

Georgia Institute of Technology

Andrés García

Georgia Institute of Technology

Nicolas Castro

Georgia Institute of Technology

\section{Eric O’Neill}

Georgia Institute of Technology

Jeroen Eyckmans

Boston University

Christopher Chen

Boston University https://orcid.org/0000-0003-2445-8449

Jianping Fu

University of Michigan-Ann Arbor https://orcid.org/0000-0001-9629-6739

David D. Schlaepfer

Moores Cancer Center, University of California San Diego

Andrés García ( $\square$ andres.garcia@me.gatech.edu )

Georgia Institute of Technology https://orcid.org/0000-0001-6602-2518

\section{Article}

Keywords: focal adhesions, FAK signaling, cell migration, tissue repair

Posted Date: October 22nd, 2020

DOI: https://doi.org/10.21203/rs.3.rs-88495/v1 
License: (c) (i) This work is licensed under a Creative Commons Attribution 4.0 International License. Read Full License

Version of Record: A version of this preprint was published at Nature Communications on April 21st, 2021. See the published version at https://doi.org/10.1038/s41467-021-22602-5. 


\section{Abstract}

How adhesive forces are transduced and integrated into biochemical signals at focal adhesions (FAs) is poorly understood. Using cells adhering to deformable micropillar arrays, we demonstrate that traction force and FAK localization as well as traction force and Y397-FAK phosphorylation are linearly coupled at individual FAs on stiff, but not soft, substrates. Similarly, FAK phosphorylation increases linearly with external forces applied to FAs using magnetic beads. This mechanosignaling coupling requires actomyosin contractility, talin-FAK binding, and full-length vinculin that binds talin and actin. Using an in vitro 3D biomimetic wound healing model, we show that force-FAK signaling coupling coordinates cell migration and tissue-scale forces to promote microtissue repair. A simple kinetic binding model of talinFAK interactions under force can recapitulate the experimental observations. This study provides insights on how talin and vinculin convert forces into FAK signaling events regulating cell migration and tissue repair.

\section{Main Text}

Mechanical forces are key regulators of development, health, and disease ${ }^{1-4}$. Focal adhesions (FAs), nanoscale complexes of structural and signaling molecules that link the extracellular matrix (ECM) to the cytoskeleton through integrin receptors, function as principal sites of mechanotransduction ${ }^{5,6}$. Studies with contractility inhibitors ${ }^{7}$, deformable substrates ${ }^{8}$, and laser tweezers ${ }^{9}$ have established that force regulates FA assembly and identified key molecules in these mechanoresponses ${ }^{10-14}$, yet very little is known about how forces are integrated into biochemical signals. Most of our understanding of mechanotransduction comes from population-based (e.g., Western blots) or whole cell assays (e.g., immunostaining of FAs in cells treated with contractility inhibitors) where the cell is viewed as in a uniform stress state. These analyses provide averaged metrics that may not reveal important relationships at the cell-ECM interface due to the heterogeneity of individual FAs in terms of force and composition. Here, we examined the relationship between force and FA signaling at individual FAs by quantifying the localization and Y397 phosphorylation of focal adhesion kinase (FAK) for cells adhering to micropost-array detectors (mPADs) ${ }^{15}$. FAK is an essential non-receptor tyrosine kinase that transduces crucial signals from FAs to regulate diverse cellular activities including survival, migration, and mechanosensing ${ }^{16}$. FAK is recruited to FAs via its FAT domain where it binds the cell membrane and other FA proteins such as talin and paxillin to become catalytically active in a multi-step process ${ }^{17}$. Autoinhibited FAK dimers bind phosphatidylinositol 4,5-bisphosphate-rich membranes to disrupt the autoinhibitory interaction between the FERM and kinase domains and expose the autophosphorylation site tyrosine 397 (Y397) for trans-autophosphorylation. Once phosphorylated, FAK functions as a molecular scaffold to recruit Src kinases to phosphorylate FAK on tyrosines 576 and 577 and become catalytically active. The autophosphorylation of FAK on Y397 is a critical early step in adhesion signaling ${ }^{18,19}$. We show that traction force and FAK localization as well as traction force and Y397-FAK phosphorylation are linearly coupled at individual FAs on stiff, but not soft, substrates. Furthermore, using 
magnetic beads to apply external forces to FAs and an in vitro 3D biomimetic wound healing model, we demonstrate that force-FAK signaling coupling coordinates mechanosensing and microtissue repair.

\section{Linear Force-FAK signaling coupling at FAs}

Mouse embryonic fibroblasts (MEFs) were cultured overnight on fibronectin-coated mPADs of either soft ( $5 \mathrm{kPa}$ ) or stiff ( $14 \mathrm{kPa}$ ) elastic modulus. A significant advantage of mPADs over traction force microscopy methods using deformable bulk gels is that the deflection for a particular post is independent from deflections of other posts, allowing isolation of forces and signaling events to the FA of interest. Because cell fixation can affect pillar deflections, we developed an optimized fixation protocol that preserves traction forces for cells on mPADs (Supplementary Fig. 1). Cells on mPADs were fixed and immunostained for total FAK (tFAK) and FAK phosphorylated at Y397 (pY397-FAK) at FAs (Fig. 1a-e, Supplementary Fig. 2). The integrated intensity (sum of intensities over the FA) for tFAK and pY397-FAK was quantified by confocal microscopy at individual FAs adhering to microposts with known deflections. In this fashion, we constructed spatial heat maps of force and tFAK (Fig. 1f,h) and pY397-FAK (Fig. 1g,i) integrated intensity for individual FAs on soft and stiff mPADs. Cells generate lower average traction forces at FAs on soft substrates (Fig. 1j) compared to stiff substrates $(P<0.0001$, Fig. $1 \mathrm{~m})$. Treatment with the ROCK inhibitor Y-27632 to impair myosin contractility significantly reduces traction forces on both soft $(P<0.0001$, Fig. 1j) and stiff $(P<0.0001$, Fig. 1m) substrates, whereas treatment with the FAK kinase inhibitor, $P F-228$, has no effect on traction forces on either soft $(P=0.4013$, Fig. 1j) or stiff $(P>0.9999$, Fig. $1 \mathrm{~m})$ substrates. No differences in average levels of tFAK $(P=0.6526$, Fig. $1 \mathbf{k}, \mathbf{n})$ or $p Y 397-F A K(P=0.1655$, Fig. 11,0) at FAs were observed between soft and stiff substrates, but treatment with either Y-27632 or PF228 reduced both tFAK (soft: $Y-27632 \mathrm{P}=0.0032$, PF-228 P=0.0195, Fig. 1k, stiff: $Y-27632 P<0.0001, P F-$ $288 \mathrm{P}=0.0004$, Fig. 1n) and pY397-FAK (soft: $Y-27632 \mathrm{P}=0.0002$, PF-228 $P<0.0001$, Fig. 11; stiff: $Y-27632$ $P<0.0001$, PF-228 $P=0.0146$, Fig. 10) levels at FAs compared to controls. These results are consistent with reports that cells generate higher traction forces, which are dependent on actomyosin contractility but not FAK kinase activity ${ }^{15,20}$, on stiff compared to soft substrates and that inhibition of actomyosin contractility reduces FAK localization and $Y 397$ phosphorylation at FAs ${ }^{12,20,21}$. Notably, the spatial heat maps for cells adhering to stiff mPADs show that FAs with high forces also have high levels of tFAK and pY397-FAK (Fig. 1h,i). We therefore plotted traction force vs. tFAK (Fig. 1p,r) and traction force vs. pY397FAK (Fig. 1q,s) for individual FAs for soft and stiff mPADs. Strikingly, tFAK and pY397-FAK levels both increase linearly with traction force at FAs for cells on stiff substrates (linear regression force-tFAK $P<0.0001$; force-pY397-FAK $P<0.0001$, Fig. 1r,s), but there is no relationship between force and tFAK $(P=0.1230)$ or force and pY397-FAK $(P=0.1072)$ on soft substrates (Fig. 1p,q). Treatment with either $Y$ 27632 or PF-228 disrupts the linear relationship on stiff substrates for force-tFAK (Fig. 1r, Y-27632 $\mathrm{P}=0.8896, \mathrm{PF}-228 \mathrm{P}=0.2084$ ) and force-pY397-FAK (Fig. 1s, Y-27632 P=0.7986, PF-228 P=0.2260), indicating that actomyosin contractility and FAK catalytic activity are required for linear force-FAK signaling coupling at FAs. The linear relationships for force-tFAK and force-pY397-FAK were observed across multiple cells (Supplementary Fig. 3 ) as well as in human mesenchymal stem cells (Supplementary Fig. 4). In addition, fibroblasts adhering to $25 \mathrm{kPa}$ mPADs also display linear force-FAK 
signaling coupling (Supplementary Fig. 5). Furthermore, treatment with the Src-family kinase inhibitor PP2 does not alter traction forces compared to controls $(P=0.2541)$ and does not disrupt the force-tFAK and force-pY397-FAK linear relationships (Supplementary Fig. 6).

We examined the contributions of talin, an essential FA component regulating integrin receptor activation and connection to the cytoskeleton ${ }^{22-24}$, to force-FAK signaling coupling on stiff (14 kPa) mPADs. Treatment with talin-1 shRNA reduces talin-1 levels by $90 \%$ and total talin (talin-1 and talin-2) levels by $60 \%$ compared to control shRNA (Supplementary Fig. 7). Talin-1 knock-down clearly impacts traction forces, tFAK levels, and pY397-FAK levels at FAs (Fig. 2a-d). Talin-1 depletion reduces total traction force (Fig. 2e, $P=0.0005$ ) and average levels of tFAK (Fig. 2f, $P<0.0001$ ) and pY397-FAK (Fig. $\mathbf{2 g}, P<0.0001$ ) at FAs as well as cell spreading area ( $P=0.0014$, Supplementary Fig. 7), consistent with previous reports ${ }^{14}$. Importantly, talin-1 depletion eliminates the linear relationship for force-tFAK (Fig. $2 \mathrm{~h}, \mathrm{P}=0.6061$ ) and force-pY397-FAK (Fig. 2i, $P=0.2421$ ). Although we cannot conclude whether talin-2 compensates for talin1 depletion, these results demonstrate that talin-1 depletion significantly reduces traction forces, FAK localization and Y397 phosphorylation and, importantly, that talin-1 is required for linear force-FAK signaling coupling at individual FAs.

We next evaluated the role of FAK functional sites on force-FAK signaling coupling using FAK-null fibroblasts expressing eGFP-FAK wild-type (WT) and mutant constructs (Fig. 2j) on stiff (14 kPa) mPADs. Expression of WT and these mutant FAK proteins in FAK-null cells increases total traction force and spreading area compared to FAK-null controls (Supplementary Fig. 8). The FAK mutants localize to FAs to equivalent levels as WT FAK (Supplementary Fig. 9), except for the K454R kinase-dead mutant which has $\sim 25 \%$ higher average levels than WT $(P=0.0004)$ and the E1015A talin-binding mutant $(P=0.0005)$. Cells expressing WT and E1015A exhibit equivalent average levels of pY397-FAK, whereas cells expressing the K454R and Y397F phosphorylation mutant display very low levels of pY397 staining (Supplementary Fig. 9). We examined the relationship between traction force and tFAK as well as traction force and pY397FAK at individual FAs. Consistent with results for wild-type MEFs (Fig. 1r,s), WT FAK-expressing FAK-null cells exhibit force-tFAK and force-pY397-FAK linear relationships (Fig. 2k,l, linear regression force-tFAK $\mathrm{P}<0.0001$; force-pY397-FAK $\mathrm{P}<0.0001)$. In contrast, FAK-null cells expressing the talin-binding mutant FAK E1015A do not show linear coupling between force and tFAK (Fig. $2 \mathrm{~m}, \mathrm{P}=0.0607$ ) and force and pY397FAK (Fig. 2n, $P=0.0619$ ) at individual $F A s$, even though the average levels of total traction force $(P=0.0721)$, tFAK localization ( $P>0.9999)$, and pY397-FAK $(P=0.2785)$ are not different between WT and E1015A FAK-expressing cells (Supplementary Fig. 8, 9). This result demonstrates that FAK binding to talin is required for linear force-FAK signaling coupling at individual FAs and is fully consistent with the requirement of talin-1 for this mechanosensing response (Fig. $2 \mathrm{~h}, \mathrm{i})$. FAK-null cells expressing the FAK K454R kinase-dead or the Y397F phosphorylation mutants show a linear relationship between force and tFAK (Fig. 20, $P<0.0001$; Fig. 2q, Y397F P<0.0001), indicating that neither FAK catalytic activity nor phosphorylation of Y397 is necessary for force-tFAK linear coupling at individual FAs. This result differs from the results with the PF-228 inhibitor (Fig. 2n). Because PF-228 may have off-target effects ${ }^{25}$, we conclude that FAK kinase activity is not required for tFAK localization to FAs based on the results for cells 
expressing the K454R mutant. However, the K454R kinase-dead mutant shows background levels of pY397-FAK (Fig. 2p), indicating that FAK kinase activity is necessary for phosphorylation of Y397 at FAs. As expected, cells expressing the Y397F phosphorylation mutant display background levels of pY397-FAK (Fig. 2r).

The requirements for substrate stiffness and actomyosin contractility in force-tFAK and forcepY397-FAK linear coupling at individual FAs suggest that a mechanical balance between traction forces and cytoskeletal tension controls force-FAK signaling coupling at FAs. We therefore analyzed the role of vinculin, an important force-transmitting protein that binds talin and actin at FAs ${ }^{24}$, in force-FAK signaling coupling using vinculin-null fibroblasts expressing WT and mutant vinculin proteins (Fig. 3a). Expression of WT vinculin in vinculin-null cells increases the average traction force at FAs compared to control vinculin-null cells $(P=0.0138)$ and cells expressing a truncated vinculin head $(\mathrm{VH})$ mutant that localizes to FAs but cannot bind actin ${ }^{24}(P<0.0001)$ or the talin binding-deficient A50l full-length mutant ${ }^{26}(P=0.0058$, Fig. 3b). No differences in average levels of tFAK (Fig. 3c) at FAs were detected between WT vinculinexpressing cells and cells expressing the $\mathrm{VH}(\mathrm{P}>0.9999)$ or $\mathrm{A} 50 \mathrm{I}$ mutant $(\mathrm{P}=0.2265)$, although higher levels of tFAK were present at FAs for vinculin-null cells compared to cells expressing WT and VH vinculin. No differences were observed in PY397-FAK at FAs among vinculin cell lines (Fig. 3d, $P=0.2276$ ). These data confirm that, whereas vinculin modulates traction force, this protein is not required for FAK localization or phosphorylation of Y397-FAK at FAs ${ }^{27}$. Nevertheless, vinculin-null cells expressing WT vinculin exhibit force-tFAK and force-pY397-FAK linear coupling (Fig. 3e,f, linear regression force-tFAK $\mathrm{P}<0.0001$, force-pY397-FAK $\mathrm{P}<0.0001$ ). In contrast, vinculin-null controls (Fig. $\mathbf{3 g}$,h, force-tFAK $\mathrm{P}=0.7124$; force-pY397-FAK $P=0.8753$ ) and cells expressing VH (Fig. 3i,j, force-tFAK $P=0.4426$; force-pY397-FAK $P=0.5564$ ) or A50I (Fig. 3k,l, force-tFAK $P=0.8549$; force-pY397-FAK $P=0.1973$ ) mutants do not exhibit linear relationships for force-tFAK and force-pY397-FAK. These results demonstrate that a full-length vinculin molecule that binds talin and actin is required for linear force-FAK signaling coupling at FAs.

\section{FAK phosphorylation increases linearly with applied external force}

The mPADs system provides a robust and experimentally convenient platform to examine the relationship between traction force and FAK signaling at individual FAs on deformable substrates with defined mechanical properties. Nevertheless, the linear relationships for force-tFAK and force-pY397-FAK on stiff substrates are generated from correlative data based on mPAD post deflections and immunostaining. To gain further insights on force-FAK signaling coupling at FAs, we used fibronectincoated magnetic beads ( $4.5 \mu \mathrm{m}$ diameter) to apply external forces to integrin-based FAs and isolate loaded adhesive complexes ${ }^{28,29}$ (Fig. 4a). After incubating adherent cells with fibronectin-coated magnetic beads for 40 minutes, a permanent magnet was placed at a defined height over the cells for 10 minutes to apply external tensile forces to FAs associated with the magnetic beads. After cell lysis, magnetic beads and associated adhesive complexes were isolated by magnetic separation, dissociated, and analyzed for tFAK and pY397-FAK levels by Western blot. Cells held in suspension for 1 hour prior to Western blot analyses served as negative controls with background levels of pY397-FAK. We first analyzed FAK-null fibroblasts expressing FAK constructs. For cells expressing WT FAK, incubation of 
beads with no applied magnetic force results in a significant increase in pY397-FAK/tFAK levels compared to suspension cells (Fig. 4b, $\mathrm{P}=0.0013$ ). Importantly, the levels of pY397-FAK/tFAK increase linearly with applied magnetic force (Fig. $\mathbf{4 b}$, linear regression $\mathrm{P}=0.0489$ ). As expected, FAK-null cells have background levels of pY397FAK/FAK (Fig. 4c). For FAK-null cells expressing the E1015A talin-binding FAK mutant, incubation of beads with no applied magnetic force results in a significant increase in pY397FAK/tFAK levels compared to suspension cells (Fig. $4 d, P=0.0158)$. However, there is no relationship between applied magnetic force and pY397-FAK/tFAK levels (Fig. 4d, $P=0.0578$ ).

We next examined the relationship between applied force and Y397-FAK phosphorylation in vinculin-null fibroblasts expressing vinculin constructs using the magnetic bead platform. For vinculinnull cells expressing WT vinculin, pY397-FAK/tFAK levels increased linearly with applied force (Fig. 4e, linear regression $P=0.0288$ ). In contrast, vinculin-null cells and vinculin-null cells expressing the $A 50$ I vinculin mutant exhibit reduced pY397-FAK/tFAK levels and applied magnetic force has no effect on pY397-FAK/tFAK levels (Fig. 4f, $P=0.4776$; Fig. 4g, $P=0.5468$ ). Taken together, these results with the magnetic bead platform demonstrate that Y397-FAK phosphorylation levels increase linearly with applied force and talin-FAK binding and vinculin are required for this linear coupling. Importantly, these results are in excellent agreement with the data obtained in the mPADs system. A limitation of the mPADs system is that FA area is limited to the size of the micropillar post (1.8 $\mu \mathrm{m}$ diameter). The magnetic bead $(4.5 \mu \mathrm{m}$ diameter sphere) provides a 12 -fold increase in available area for FAs, and the high concordance in results between these two platforms suggests that the linear force-FAK signaling coupling is not limited to the small available area of mPADs.

\section{Force-FAK signaling coupling coordinates microtissue repair}

To investigate the biological significance of force-FAK signaling coupling, we employed an in vitro biomimetic wound healing model using microfabricated tissue constructs ${ }^{30}$ (Fig. 5a). In this platform, a 3D collagen gel seeded with cells is suspended between flexible cantilevers within a microfabricated mold, and encapsulated cells generate traction forces to contract the collagen gel into a dense fibrocellular microtissue attached to the deformable posts. A microsurgically-induced defect (i.e., wound) in the center of the microtissue is rapidly closed by coordinated cell forces and migration, providing an in vitro model of 3D fibrous tissue repair. We examined the tissue repair response for FAK-null fibroblasts expressing WT FAK or the E1015A talin-binding FAK mutant or control FAK-null cells (Supplementary movies 1-3). For microtissues containing WT FAK-expressing cells, the wound area rapidly increases following injury due to pre-stress arising from cell force-driven tissue compaction, but wound area then decreases monotonically as the tissue repairs until it is fully closed (Fig. 5b). The profile for wound area, normalized to initial wound area, over time is accurately described by a log-normal curve (Fig. 5b), and this curve fit was used to estimate amplitude and wound closure rate parameters to describe the tissue healing response (Supplementary Fig. 10). In stark contrast to WT FAK-expressing cells, wounds for microtissues containing FAK-null cells maintain constant area over time and do not close (Fig. 5b), demonstrating that FAK is required for microtissue repair. Microtissues containing cells expressing the E1015A FAK mutant exhibit delayed wound closure and several microtissues did not fully repair wounds 
(Fig. 5b). Analysis of curve-fit parameters confirms these observations. Microtissues containing WT FAKexpressing cells exhibit higher amplitude, reflecting higher pre-stress prior to injury, compared to microtissues seeded with E1015A FAK-expressing cells ( $P=0.0440)$ and FAK-null cells (Fig. 5c, $P=0.0176$ ). Furthermore, microtissues containing WT FAK-expressing cells display faster closure rates compared to microtissues seeded with E1015A FAK-expressing cells ( $P=0.0213)$ and FAK-null cells (Fig. $5 d, P=0.0088$ ). The wound closure rate for microtissues seeded with E1015A FAK-expressing cells is higher than the rate for tissues with FAK-null cells $(P=0.0289)$. At 24 hours post-wounding, microtissues seeded with WT FAKexpressing cells generate higher contractile forces compared to microtissues containing E1015A FAKexpressing cells $(\mathrm{P}=0.0266)$ and FAK-null cells (Fig. 5e, $\mathrm{P}=0.0003)$. In addition, microtissues containing WT FAK- or E1015A FAK-expressing cells exhibit higher width contraction along the centerline compared to microtissues seeded with FAK-null cells (Fig. 5f, $P=0.0015, P=0.0101$ ). Microtissues containing WT FAK-expressing cells display higher levels of pY397-FAK intensity compared to microtissues seeded with E1015A FAK-expressing cells ( $P=0.0232)$ and FAK-null cells (Fig. $\mathbf{5 g}, \mathrm{P}=0.0020)$. These results demonstrate that FAK expression and FAK-talin binding are required for tissue-scale force generation and proper microtissue repair. Consistent with this conclusion, microtissues containing talin-1-depleted cells exhibit reduced amplitude values, indicating reduced tissue pre-stress, and impaired wound closure rates compared to microtissues seeded with control cells (Supplementary Fig. 11).

Chen and colleagues demonstrated that microtissue wound closure requires coordinated cell migration ${ }^{30}$. Using automated cell tracking and image analysis, we measured individual cell trajectories during microtissue wound healing (Supplementary Fig. 12). Mean square displacement (MSD), cell speed, and straightness index (directionality index) were calculated from individual cell trajectories using MotilityLab (Supplementary Fig. 12). This analysis reveals lower migration speed and straightness index for WT FAK-expressing cells compared to FAK-null cells $(P<0.0001, P<0.0001)$ and cells expressing the E1015A FAK mutant $(P=0.0003, P=0.0015)$. Speed and straightness parameters are also different between E1015A FAK-expressing and FAK-null cells $(P=0.0025, P=0.0051)$. Furthermore, the MSD data were curve fit to the Persistent Random Walk model to extract values for diffusivity and directional persistence. WT FAK-expressing cells display reduced persistence and diffusivity values compared to E1015A FAK-expressing ( $P=0.0202, P=0.0284)$ and FAK-null $(P=0.0009, P=0.0006)$ cells. Taken together, these migration parameters show that WT FAK-expressing cells in microtissues exhibit slower and more directed cell motions compared E1015A FAK-expressing and FAK-null fibroblasts in microtissues. These results demonstrate that FAK-talin binding is required for coordinated and directed cell migration for microtissue wound closure.

\section{Model of talin-FAK binding under force}

We developed a simple kinetic model to simulate talin-FAK binding interactions under force to gain further insights into force-FAK signaling coupling (Fig. 6a). In this model, talin at FAs undergoes a reversible structural change from an unstretched to a stretched conformation under force. The effect of tension across talin is incorporated into the kinetic rate controlling talin conversion into the stretched state using the Bell model ${ }^{31}$ and in agreement with experimental measurements of talin stretching under force ${ }^{13}$. The 
stretched talin reversibly binds FAK to form a FAK-talin complex, and FAK in the FAK-talin complex then undergoes reversible phosphorylation. The law of mass action and conservation laws were applied to derive a system of coupled differential equations describing the time-dependent changes for the number of stretched talin molecules, FAK-talin complexes, and phosphorylated FAK-talin complexes. Solutions at steady state were obtained numerically as a function of force applied to talin over a range of forces consistent with measurements in live cells $(7-11 \mathrm{pN})^{22,32}$, and parametric analyses were performed for relevant conditions. We first explored the influence of the coupling factor a that relates the force applied to talin to the forward rate controlling conversion into the talin stretched state $\left(k_{1}\right)$. For $a=0.05$, a value in agreement with experimental measurements of talin unfolding under force ${ }^{13}$, the number of stretched talin molecules, FAK-talin complexes, and phosphorylated FAK-talin complexes increase linearly with force applied to talin (Fig. $\mathbf{6 b - d}$ ). The predicted linear increases in FAK-talin complexes and phosphorylated FAK-talin complexes with force are in good agreement with our experimental observations for the linearity of force-FAK signaling coupling at individual FAs on stiff substrates (Fig. $1 \mathrm{r}, \mathbf{s})$. For smaller values of $\mathrm{a}$, the number of stretched talin molecules, FAK-talin complexes, and phosphorylated FAK-talin complexes are independent of the force applied to talin. These simulations are in line with our experimental results showing that inhibition of actomyosin contractility (Fig. 1r,s) or adhesion to soft substrates (Fig. 1p,q) eliminates the linear relationship for force-FAK signaling at FAs. Smaller values of a correspond to kinetic rates of switching between unstretched and stretched talin conformations that are insensitive to force, and therefore reflect a talin molecule that does not respond to force. Consistent with this explanation, Grashoff and Schwartz independently reported that inhibition of contractility or adhesion to soft substrates reduces tension across talin 22,32 .

Our experimental findings demonstrate that the talin-binding site in FAK is required for linear force-FAK signaling coupling (Fig. 2m,n), mechanosensing (Fig. 4d), and microtissue repair (Fig. 5b-e). Mutation of this site can be modeled as a reduction in $\mathrm{k}_{3}$, the forward binding rate for the FAK-talin complex. Computer simulations show that decreases in the value of $k_{3}$ considerably reduce the number of FAKtalin complexes and phosphorylated FAK-talin complexes (Fig. 6f,g), and a 100-fold reduction in $\mathrm{k}_{3}$ eliminates the dependence of the number of phosphorylated FAK-talin complexes on force applied to talin. We conducted additional simulations to examine the effect of the FAK phosphorylation rate $k_{5}$. As expected, decreases in $k_{5}$ drastically reduce the number of phosphorylated FAK-talin complexes and eliminate its dependence on force, but decreases in $\mathbf{k}_{5}$ have relatively modest effects on the linear relationship between applied force and the number of stretched talin molecules and FAK-talin complexes (Fig. 6h-j). These simulations mirror the experimental results for cells expressing the FAK K454R kinasedead mutant showing a linear relationship between traction force and tFAK and background levels of pY397-FAK (Fig. 20,p).

The kinetic binding model does not explicitly consider vinculin's role in linear force-FAK signaling coupling. Based on the requirement of the talin-binding site in vinculin for linear force-FAK signaling coupling (Fig. 3k,l), we posit that vinculin binds to and stabilizes the stretched talin conformation while transmitting force to actin. As such, the contributions of vinculin can be lumped within the kinetic rates of 
switching between unstretched and stretched talin conformations. Several lines of evidence support this idea. First, tension across talin exposes cryptic binding sites for vinculin binding ${ }^{13}$. Second, tension across talin at FAs is decreased in the absence of vinculin but re-expression of full-length vinculin restores mechanical loading of talin ${ }^{22}$. Interestingly, a full-length vinculin that binds talin and actin is needed for both mechanical loading of talin ${ }^{22}$ and linear force-FAK signaling coupling (Fig. 3e,f).

\section{Outlook}

We demonstrate linear coupling between traction force and FAK localization as well as traction force and Y397-FAK phosphorylation at individual FAs on stiff, but not soft, substrates. Linear force-FAK signaling coupling requires actomyosin contractility, talin binding to FAK, and vinculin capable of binding both talin and actin. Furthermore, FAK phosphorylation increases linearly with applied external force, and talin binding to FAK and vinculin are required for this dose-dependent response. Using a biomimetic 3D model of wound closure, we show that vinculin and talin-FAK interactions are necessary for tissue-scale forces and effective wound closure. A simple kinetic binding model of talin-FAK binding interactions under force can recapitulate the experimental observations. Our model proposes force-dependent structural changes in talin that regulate its interaction with FAK but does not consider direct effects of force on FAK. Lietha et al. reported that force induces structural changes in FAK to pull the kinase domain away from the FERM domain $^{33}$. While we cannot rule out a direct role of force on FAK activation, we speculate that the signaling events examined here occur after FAK adopts an open conformation on the cell membrane because the E1015A FAK mutant is still able to localize to FAs and become phosphorylated at Y397.

These experimental and modeling analyses provide insights on how talin, FAK, and vinculin convert forces into early signaling events regulating mechanotransduction. This conceptual framework is relevant to adhesive force-signaling coupling at migratory cell fronts, force-regulated morphogenesis, and stem cell lineage commitment in response to matrix stiffness. Furthermore, this fundamental understanding of mechanosignaling can ultimately be exploited to design cell-biomaterial interactions. Exemplary applications include the design of biomaterial tools for basic cell biology studies, development of culture supports for therapeutic cell manufacturing, and engineering of synthetic stem cell niches for regenerative medicine.

\section{Declarations}

Acknowledgments: Authors thank B. Koehler for help with data analysis and T.H. Barker for recommendations related to magnetic bead assay. We acknowledge support from the National Institutes of Health Award No. R01 EB024322 (A.J.G.), R01 CA102310 and R01 CA247562 (D.D.S.), and R21 EB028491 (J.E.), and the National Science Foundation Graduate Research Fellowship (D.W.Z.) under Grant No. DGE-1148903 and the NSF Science and Technology Center for Engineering Mechanobiology (C.S.C.). The contents of this publication are solely the responsibility of the authors and do not necessarily represent the official views of the NIH or NSF. 
Author contributions: D.W.Z., M.A.F.-Y., N.S.C., E.N.H., E.B.O., A.J.G. conducted experiments and analyses; A.F.G. wrote and validated MATLAB code for model. A.J.G. conceptualized and supervised the research. D.D.S. provided the mutant FAK cell lines and input on interpretation of results. J.F. provided the mPADs master molds and suggestions on experimental design and data interpretation. J.E. and C.S.C. provided masters for microtissue molds and input on interpretation of results. D.W.Z., M.A.F.-Y., and A.J.G. wrote the manuscript, with contributions from all other authors.

Competing interests: The authors declare no conflicts of interest.

\section{References}

1. Wozniak MA, Chen CS. Mechanotransduction in development: a growing role for contractility. Nat Rev Mol Cell Biol 2009, 10(1): 34-43.

2. Discher DE, Mooney DJ, Zandstra PW. Growth factors, matrices, and forces combine and control stem cells. Science 2009, 324(5935): 1673-1677.

3. Chan CJ, Costanzo M, Ruiz-Herrero T, Monke G, Petrie RJ, Bergert M, et al. Hydraulic control of mammalian embryo size and cell fate. Nature 2019, 571(7763):112-116.

4. DuFort CC, Paszek MJ, Weaver VM. Balancing forces: architectural control of mechanotransduction. Nat Rev Mol Cell Biol 2011, 12(5): 308-319.

5. Vogel V, Sheetz MP. Cell fate regulation by coupling mechanical cycles to biochemical signaling pathways. Curr Opin Cell Biol 2009, 21(1):38-46.

6. Parsons JT, Horwitz AR, Schwartz MA. Cell adhesion: integrating cytoskeletal dynamics and cellular tension. Nat Rev Mol Cell Biol 2010, 11(9): 633-643.

7. Kuo JC, Han X, Hsiao CT, Yates JR, 3rd, Waterman CM. Analysis of the myosin-II-responsive focal adhesion proteome reveals a role for beta-Pix in negative regulation of focal adhesion maturation. Nat Cell Biol 2011, 13(4): 383-393.

8. Yeung T, Georges PC, Flanagan LA, Marg B, Ortiz M, Funaki M, et al. Effects of substrate stiffness on cell morphology, cytoskeletal structure, and adhesion. Cell Motil Cytoskeleton 2005, 60(1): 24-34.

9. Neuman KC, Nagy A. Single-molecule force spectroscopy: optical tweezers, magnetic tweezers and atomic force microscopy. Nat Methods 2008, 5(6): 491-505.

10. Balaban NQ, Schwarz US, Riveline D, Goichberg P, Tzur G, Sabanay I, et al. Force and focal adhesion assembly: a close relationship studied using elastic micropatterned substrates. Nat Cell Bio/ 2001, 3(5): 466-472. 
11. Riveline D, Zamir E, Balaban NQ, Schwarz US, Ishizaki T, Narumiya S, et al. Focal contacts as mechanosensors: externally applied local mechanical force induces growth of focal contacts by an mDia1-dependent and ROCK-independent mechanism. J Cell Bio/ 2001, 153(6): 1175-1186.

12. Plotnikov SV, Pasapera AM, Sabass B, Waterman CM. Force fluctuations within focal adhesions mediate ECM-rigidity sensing to guide directed cell migration. Cel/ 2012, 151(7): 1513-1527.

13. del Rio A, Perez-Jimenez R, Liu R, Roca-Cusachs P, Fernandez JM, Sheetz MP. Stretching single talin rod molecules activates vinculin binding. Science 2009, 323(5914): 638-641.

14. Elosegui-Artola A, Oria R, Chen Y, Kosmalska A, Perez-Gonzalez C, Castro N, et al. Mechanical regulation of a molecular clutch defines force transmission and transduction in response to matrix rigidity. Nat Cell Biol 2016, 18(5): 540-548.

15. Fu J, Wang YK, Yang MT, Desai RA, Yu X, Liu Z, et al. Mechanical regulation of cell function with geometrically modulated elastomeric substrates. Nat Methods 2010, 7(9): 733-736.

16. Kleinschmidt EG, Schlaepfer DD. Focal adhesion kinase signaling in unexpected places. Curr Opin Cell Biol 2017, 45: 24-30.

17. Acebrón I, Righetto RD, Schoenherr C, de Buhr S, Redondo P, Culley J, et al. Structural basis of Focal Adhesion Kinase activation on lipid membranes. EMBO J 2020, 39(19): e104743.

18. Mitra SK, Hanson DA, Schlaepfer DD. Focal adhesion kinase: in command and control of cell motility. Nat Rev Mol Cell Biol 2005, 6(1): 56-68.

19. Schlaepfer DD, Hauck CR, Sieg DJ. Signaling through focal adhesion kinase. Progress Biophys Mol Biol 1999, 71(3-4): 435-478.

20. Pasapera AM, Schneider IC, Rericha E, Schlaepfer DD, Waterman CM. Myosin II activity regulates vinculin recruitment to focal adhesions through FAK-mediated paxillin phosphorylation. $J$ Cell Biol 2010, 188(6): 877-890.

21. Lim ST, Chen XL, Tomar A, Miller NL, Yoo J, Schlaepfer DD. Knock-in mutation reveals an essential role for focal adhesion kinase activity in blood vessel morphogenesis and cell motility-polarity but not cell proliferation. J Biol Chem 2010, 285(28): 21526-21536.

22. Austen K, Ringer P, Mehlich A, Chrostek-Grashoff A, Kluger C, Klingner C, et al. Extracellular rigidity sensing by talin isoform-specific mechanical linkages. Nat Cell Biol 2015, 17(12): 1597-1606.

23. Atherton P, Stutchbury B, Wang DY, Jethwa D, Tsang R, Meiler-Rodriguez E, et al. Vinculin controls talin engagement with the actomyosin machinery. Nat Commun 2015, 6: 10038. 
24. Cohen DM, Kutscher B, Chen H, Murphy DB, Craig SW. A conformational switch in vinculin drives formation and dynamics of a talin-vinculin complex at focal adhesions. J Biol Chem 2006, 281(23): 16006-16015.

25. Slack-Davis JK, Martin KH, Tilghman RW, Iwanicki M, Ung EJ, Autry C, et al. Cellular characterization of a novel focal adhesion kinase inhibitor. J Biol Chem 2007, 282(20): 14845-14852.

26. Cohen DM, Chen H, Johnson RP, Choudhury B, Craig SW. Two distinct head-tail interfaces cooperate to suppress activation of vinculin by talin. J Biol Chem 2005, 280(17): 17109-17117.

27. Rahman A, Carey SP, Kraning-Rush CM, Goldblatt ZE, Bordeleau F, Lampi MC, et al. Vinculin regulates directionality and cell polarity in 2D, 3D matrix and 3D microtrack migration. Mol Biol Cell 2016, 27(9): 1431-1441.

28. Glogauer M, Ferrier J, McCulloch CA. Magnetic fields applied to collagen-coated ferric oxide beads induce stretch-activated Ca2+ flux in fibroblasts. Am J Physiol 1995, 269(5 Pt 1): C1093-1104.

29. Guilluy C, Swaminathan V, Garcia-Mata R, O'Brien ET, Superfine R, Burridge K. The Rho GEFs LARG and GEF-H1 regulate the mechanical response to force on integrins. Nat Cell Bio/ 2011, 13(6): 722727.

30. Sakar MS, Eyckmans J, Pieters R, Eberli D, Nelson BJ, Chen CS. Cellular forces and matrix assembly coordinate fibrous tissue repair. Nat Commun 2016, 7: 11036.

31. Bell GI. Models for the specific adhesion of cells to cells. Science 1978, 200: 618-627.

32. Kumar A, Ouyang M, Van den Dries K, McGhee EJ, Tanaka K, Anderson MD, et al. Talin tension sensor reveals novel features of focal adhesion force transmission and mechanosensitivity. J Cell Biol 2016, 213(3): 371-383.

33. Bauer MS, Baumann F, Daday C, Redondo P, Durner E, Jobst MA, et al. Structural and mechanistic insights into mechanoactivation of focal adhesion kinase. Proc Natl Acad Sci U S A 2019, 116(14): 6766-6774.

\section{Figures}


b

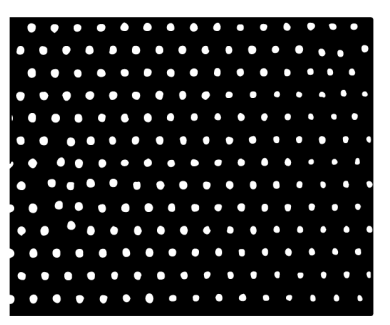

C

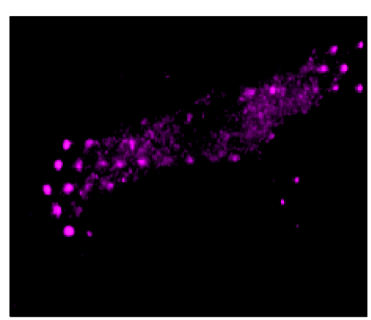

$5 \mathrm{kPa}$

$\mathbf{f}$

f $\quad$ tFAK

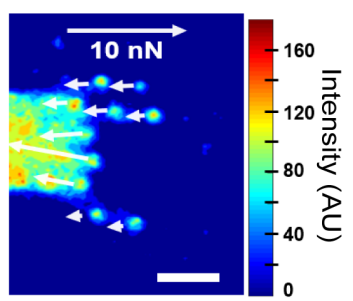

g PY397-FAK

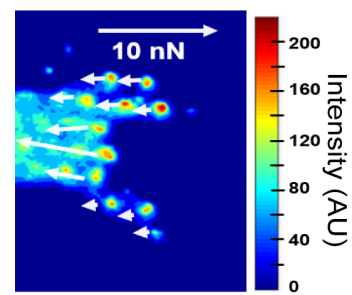

j

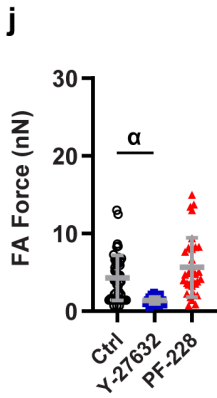

p

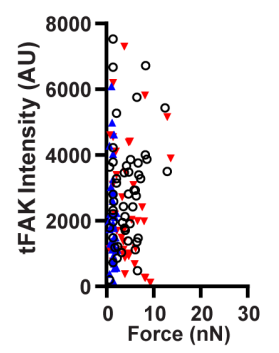

q
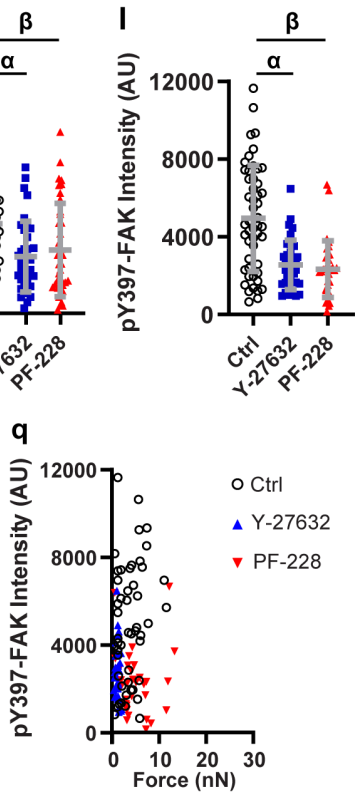

d pY397-FAK

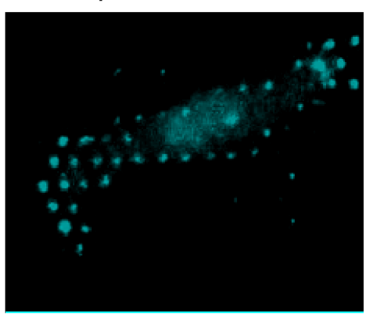

$14 \mathrm{kPa}$

h

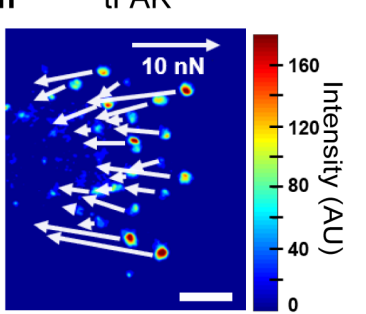

i pY397-FAK
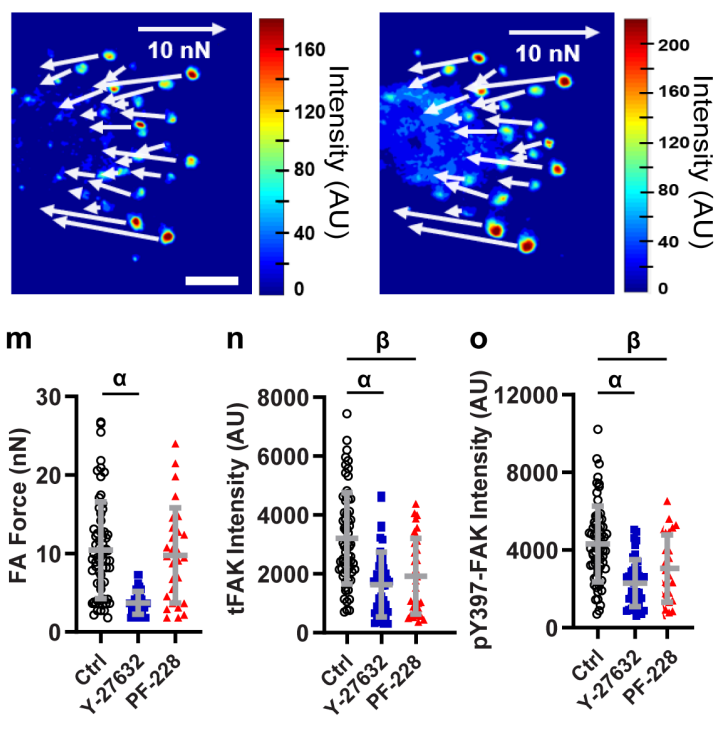

n

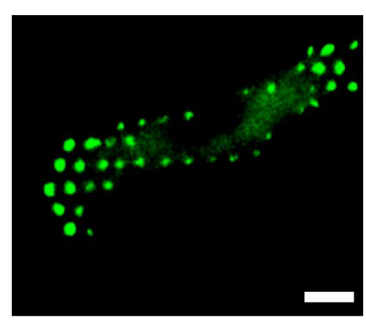

$\therefore$
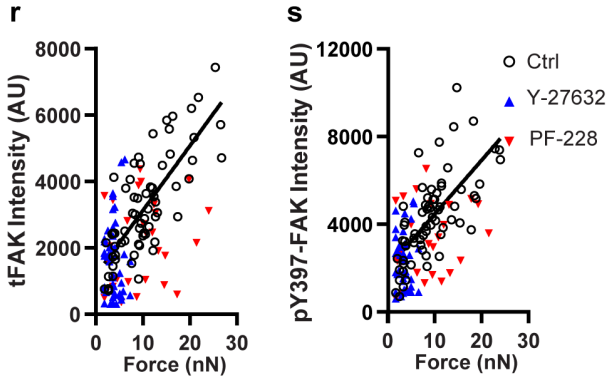

\section{Figure 1}

Force-FAK localization and force-pY397-FAK are linearly coupled at individual FAs. a, Schematic of individual FA traction force measurement for a cell using mPADs. Fluorescence images showing (b) mPAD posts, (c) tFAK, (d) pY397-FAK, and (e) eGFP-vinculin for cell on mPAD. f-i, Heatmaps for (f,h) tFAK and $(\mathrm{g}, \mathrm{i})$ pY397-FAK staining at FAs on mPADs of 5 or $14 \mathrm{kPa}$. White arrows indicate force magnitudes and directions. Scale bar $5 \mu \mathrm{m}$. j, Force (mean \pm SD) at individual FAs on 5 kPa mPADs. Kruskal-Wallis 
test $P<0.0001$, $a: P<0.0001$. $k$, tFAK integrated intensity (mean $\pm S D$ ) at FAs on $5 \mathrm{kPa}$ mPADs. ANOVA $P<0.0001$, $a: P=0.0032, \beta: P=0.0195$. I, $p Y 397-F A K$ integrated intensity (mean $\pm S D$ ) at individual FAs on 5 kPa mPADs. Kruskal-Wallis test $P<0.0001$, $a: P=0.0002, \beta$ : $P<0.0001$. $m$, Force (mean $\pm S D$ ) at individual FAs on $14 \mathrm{kPa}$ mPADs. Kruskal-Wallis test $P<0.0001, a$ : $P<0.0001$. $n$, tFAK integrated intensity (mean \pm $\mathrm{SD}$ ) at FAs on $14 \mathrm{kPa}$ mPADs. ANOVA $P<0.0001$, $a: P<0.0001, \beta$ : $P=0.0004$. o, pY397-FAK integrated intensity (mean $\pm S D$ ) at individual FAs on $14 \mathrm{kPa}$ mPADs. Kruskal-Wallis test $P<0.0001, a: P<0.0001, \beta$ : $P<0.0146$. $p$, Force vs tFAK intensity at individual FAs on $5 \mathrm{kPa}$. Linear regression: control $(P=0.1230$, $n=50$ FAs), $Y-27632$ ( $P=0.1920, n=37$ FAs), and PF-228 ( $P=0.9869, n=38$ FAs). $q$, Force vs pY397-FAK intensity at individual FAs on $5 \mathrm{kPa}$. Linear regression: control ( $P=0.1072, n=50 F A s), Y-27632$ ( $P=0.2937$, $\mathrm{n}=36 \mathrm{FAs})$, and $\mathrm{PF}-228(\mathrm{P}=0.8874, \mathrm{n}=33 \mathrm{FAs})$. $\mathrm{r}$, Force vs tFAK intensity at individual FAs on $14 \mathrm{kPa}$. Linear regression: control $\left(P<0.0001, n=66\right.$ FAs, tFAK intensity $=194.6^{*}$ force +1178$), Y-27632(P=0.8896$, $\mathrm{n}=48 \mathrm{FAs})$, and PF-228 ( $\mathrm{P}=0.2084, \mathrm{n}=28 \mathrm{FAs})$. $\mathrm{s}$, Force vs $\mathrm{pY397-FAK} \mathrm{intensity} \mathrm{at} \mathrm{individual} \mathrm{FAs} \mathrm{on} 14$ $\mathrm{kPa}$. Linear regression: control $\left(P<0.0001, n=66 \mathrm{FAs}, \mathrm{pY} 397-F A K\right.$ intensity $=219.7^{*}$ force +2027$), \mathrm{Y}-27632$ $(\mathrm{P}=0.7986, \mathrm{n}=46 \mathrm{FAs})$, and PF-228 ( $\mathrm{P}=0.2260, \mathrm{n}=26 \mathrm{FAs})$. 
Ctrl shRNA
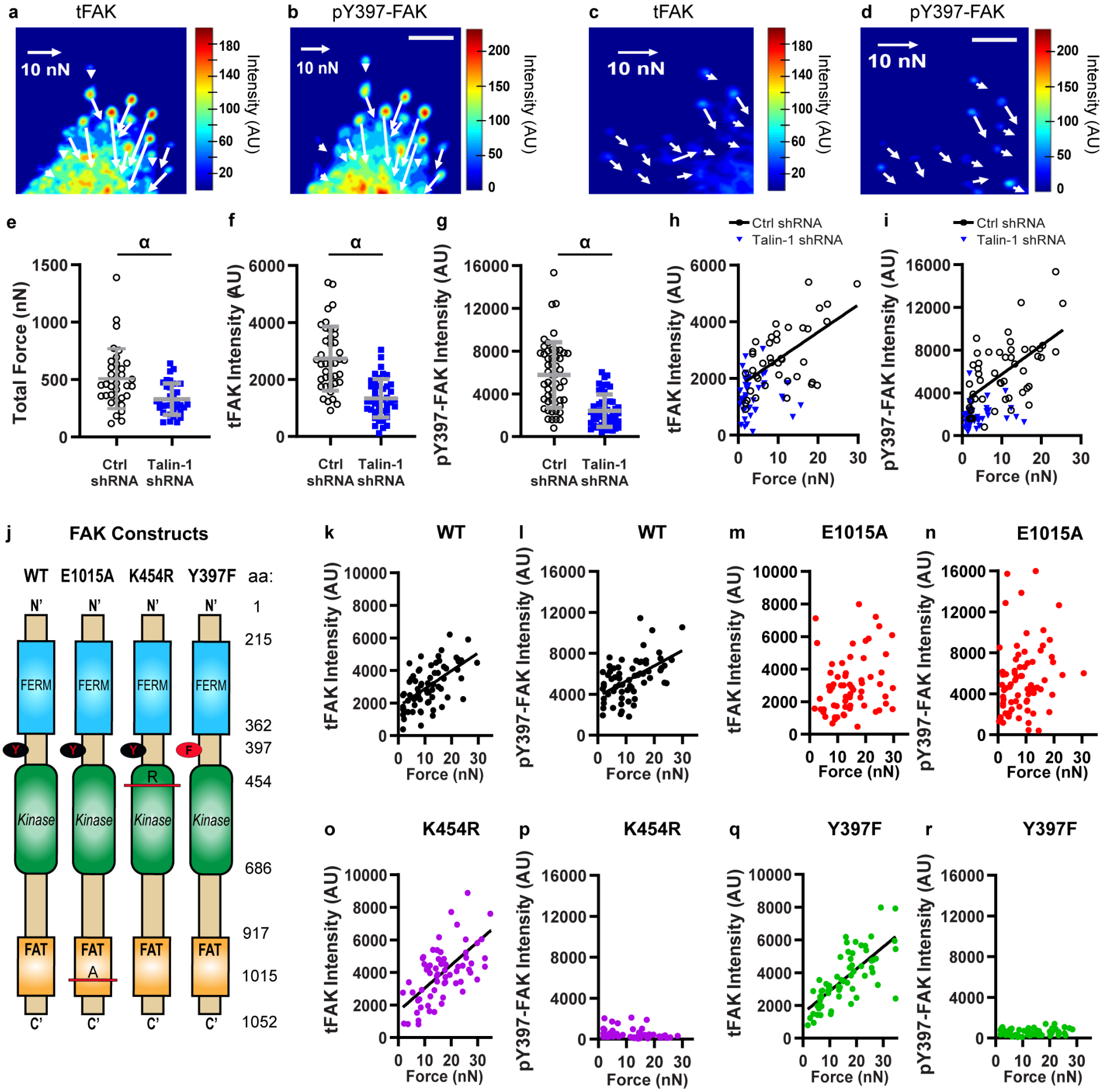

Figure 2

Talin regulates linear force-FAK signaling coupling at FAs. a-d, Heatmaps of tFAK and pY397-FAK intensities at FAs for cells treated with $(a, b)$ control or $(c, d)$ talin-1 shRNA. Scale bar $5 \mu \mathrm{m}$. e, Total traction force (mean \pm SD) for control shRNA- and talin-1 shRNA-treated cells. Mann-Whitney test $a: P=0.0005$. $f$, tFAK intensity (mean \pm SD) at individual FAs. Mann-Whitney test $a$ : $P<0.0001$. g, pY397-FAK intensity (mean \pm SD) at individual FAs. Mann-Whitney test $\mathrm{a}: \mathrm{P}<0.0001$. $\mathrm{h}$, Traction force vs tFAK intensity at individual FAs. Linear regression: control shRNA $(P<0.0001, n=40$ FAs, tFAK intensity $=96.1 *$ force + 
1712), talin-1 shRNA ( $P=0.6061, n=42$ FAs). i, Force vs pY397-FAK intensity at individual FAs. Linear regression: control shRNA ( $P<0.0001, n=51$ FAs, pY397-FAK intensity $=268.3^{\star}$ force +3056$)$, talin-1 shRNA $(P=0.2421, n=42 F A s)$. j, Schematic of FAK constructs. $k$, Force vs tFAK intensity at individual FAs for WT FAK. Linear regression $\left(P<0.0001, n=70\right.$ FAs, tFAK intensity $=110.2^{\star}$ force +1779$)$. I, Force vs pY397-FAK intensity at individual FAs for WT FAK. Linear regression $(P<0.0001, n=68$ FAs, pY397-FAK intensity $=$ 150.0*force + 3776). $m$, Force vs tFAK intensity at individual FAs for E1015A FAK. Linear regression $(\mathrm{P}=0.0607, \mathrm{n}=65 \mathrm{FAs}) . \mathrm{n}$, Force vs pY397-FAK intensity at individual FAs for E1015A FAK. Linear regression ( $P=0.0619, n=78$ FAs). o, Force vs tFAK intensity at individual FAs for K454R FAK. Linear regression $\left(P<0.0001, n=70\right.$ FAs, tFAK intensity $=142.1^{\star}$ force +1607$)$. $p$, Force vs pY397-FAK intensity at individual FAs for K454R FAK. Linear regression ( $P=0.0610$, $n=48$ FAs). $q$, Force vs tFAK intensity at individual FAs for Y397F FAK. Linear regression $\left(P<0.0001, n=65\right.$ FAs, tFAK intensity $=135.1^{\star}$ force + 1525). r, Force vs $p Y 397-F A K$ intensity at individual FAs for Y397F FAK. Linear regression $(P=0.1869, n=60$ FAs).
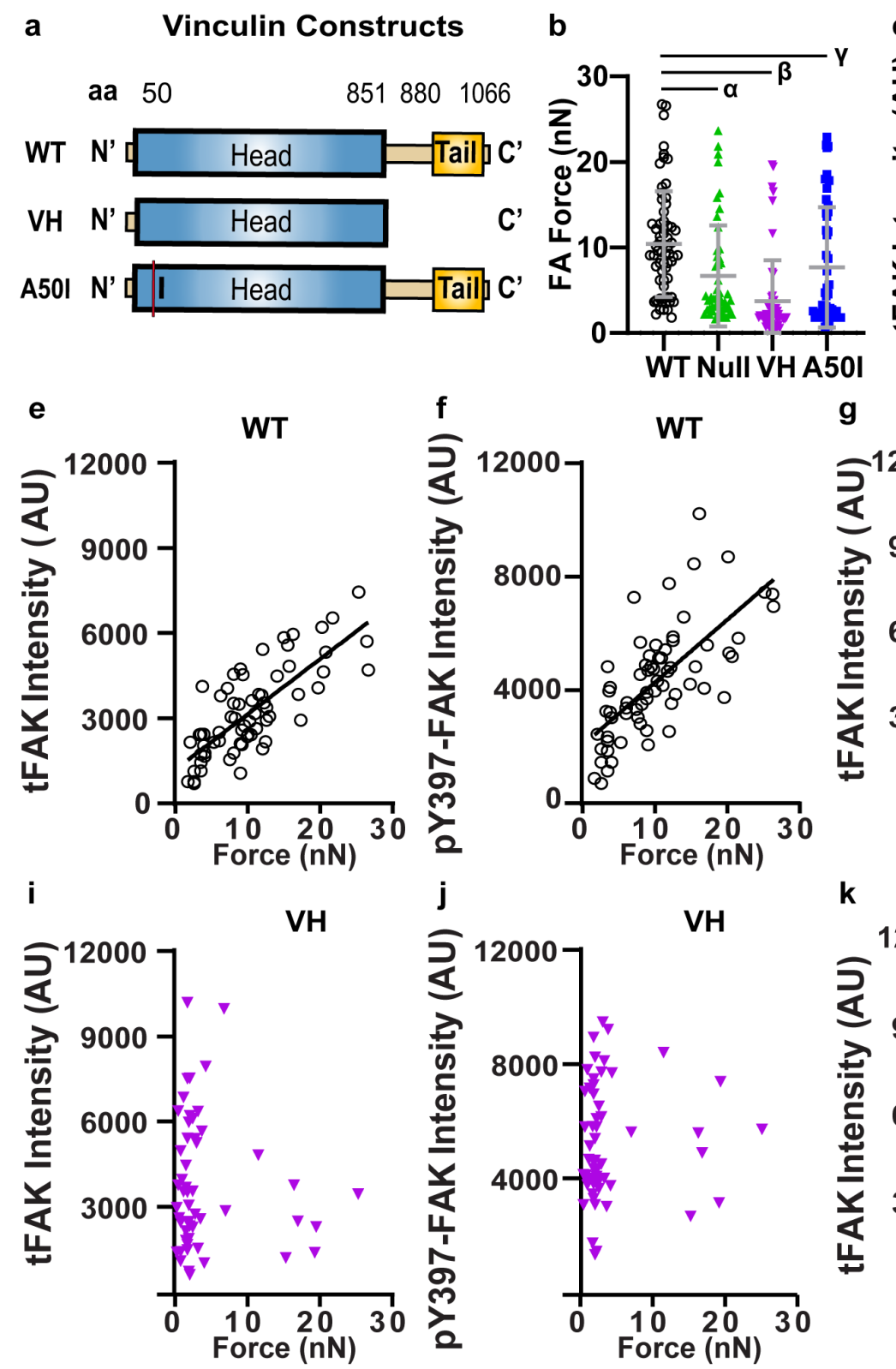

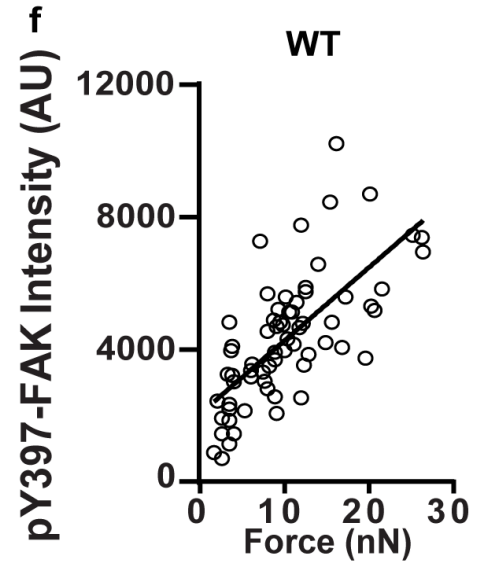

g
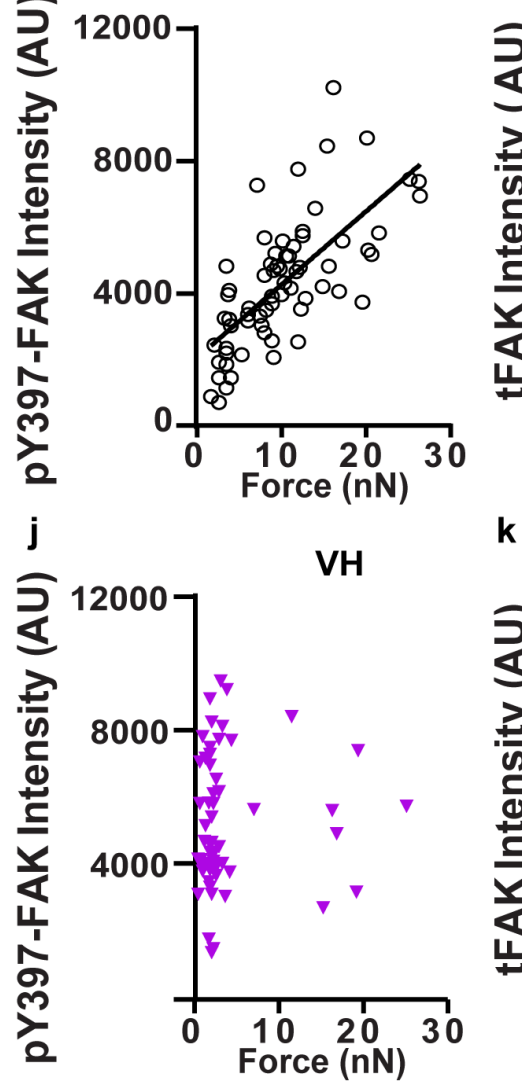

k
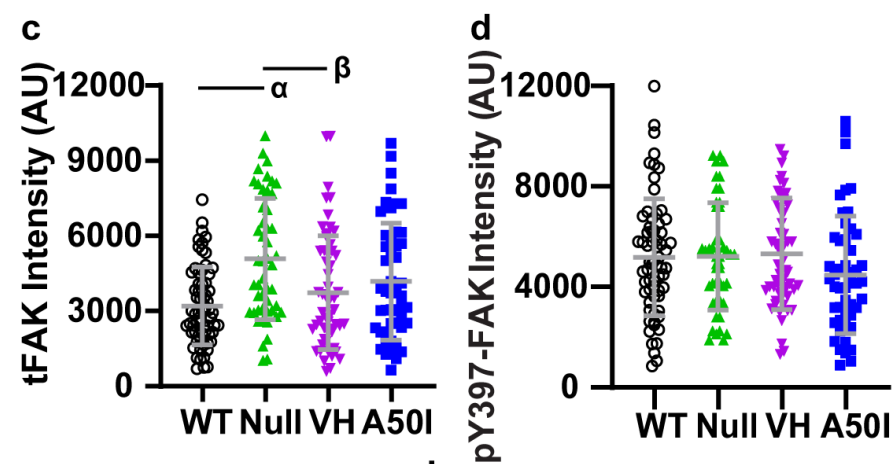

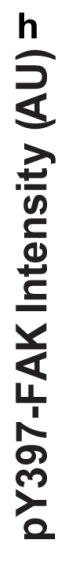

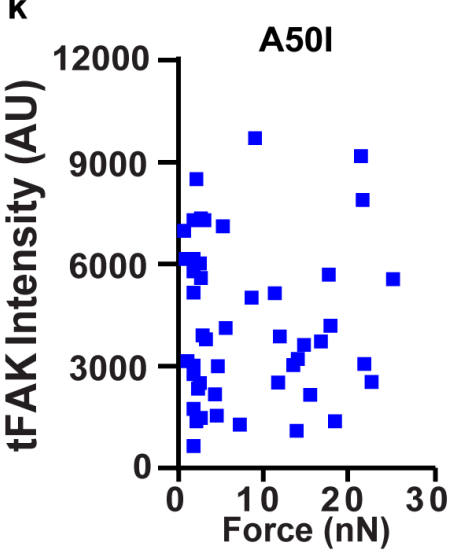

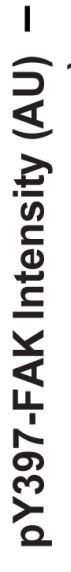

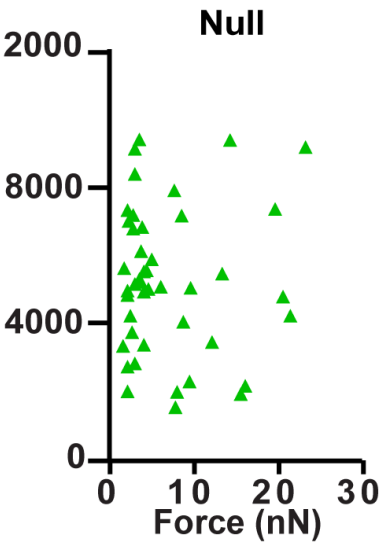

A50I

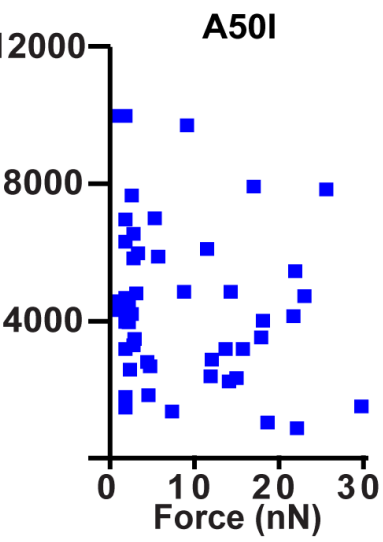

Figure 3 
Vinculin mediates linear force-FAK signaling coupling at FAs. a, Vinculin constructs. b, Traction force (mean \pm SD) at individual FAs. Kruskal-Wallis test $P<0.0001, a: P=0.0138, \beta: P<0.0001, y: P=0.0058$. $c$, tFAK intensity (mean \pm SD) at individual FAs. Kruskal-Wallis test $P=0.0003, a: P=0.0002, \beta: P<0.0084$. $d$, pY397-FAK intensity (mean \pm SD) at individual FAs. ANOVA P=0.2276. e, Force vs tFAK intensity at individual FAs for WT vinculin. Linear regression $(P<0.0001, n=66$ FAs, tFAK intensity $=194.6 *$ force + 1178). f, Force vs pY397-FAK intensity at individual FAs for WT vinculin. Linear regression $(P<0.0001$, $\mathrm{n}=66$ FAs, pY397-FAK intensity $=219.7 *$ force +2027$)$. g, Force vs tFAK intensity at individual FAs for vinculin-null cells. Linear regression ( $P=0.7124, n=49 F A s)$. h, Force vs pY397-FAK intensity at individual FAs for vinculin-null cells. Linear regression ( $P=0.8753, n=48$ FAs). i, Force vs tFAK intensity at individual FAs for VH vinculin. Linear regression ( $P=0.4426, n=57$ FAs). j, Force vs pY397-FAK intensity at individual FAs for VH vinculin. Linear regression ( $P=0.5564, n=57$ FAs). $k$, Force vs tFAK intensity at individual FAs for $A 50$ I vinculin. Linear regression ( $P=0.8549, n=49$ FAs). I, Force vs $p Y 397-F A K$ intensity at individual FAs for A50I vinculin. Linear regression ( $P=0.1973, n=49$ FAs).
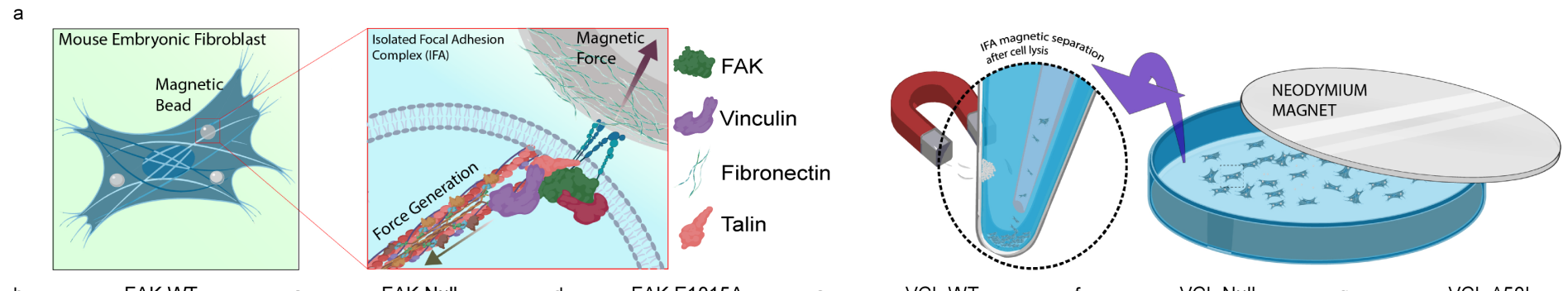

$\mathrm{b}$
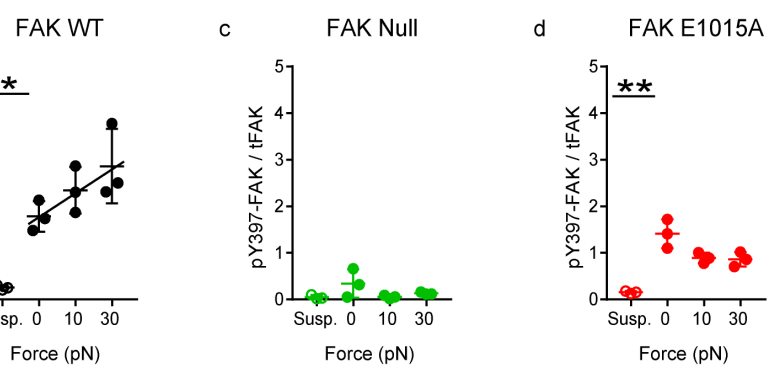

e
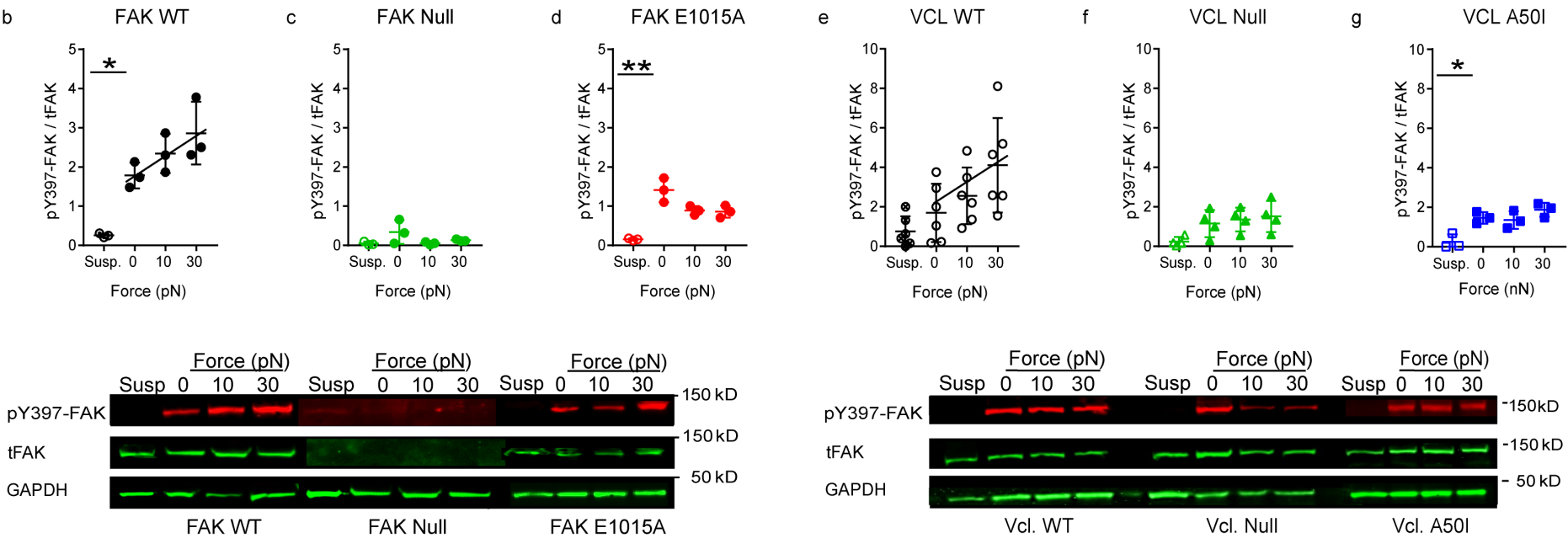

Figure 4

FAK phosphorylation increases linearly with applied force. a, Schematic of application of external force to integrin-based FAs and isolation of FAs using magnetic beads. b-d, pY397-FAK/tFAK levels for FAK-null cells expressing FAK constructs (top) and Western blots (below). b, For WT FAK-expressing cells, pY397FAK/tFAK levels (mean $\pm S D$ ) increase linearly with applied force. Linear regression $(P=0.0489, p Y 397-$ FAK/FAK $=0.0345^{\star}$ force +1.870$)$. t-test $a: P=0.0013$. c , For FAK-null cells, background levels of pY397FAK/tFAK (mean \pm SD) are detected. $d$, For cells expressing FAK E1015A, pY397-FAK/tFAK levels (mean \pm $S D)$ are independent of external force. Linear regression ( $P=0.0578)$. t-test $a: P=0.0158$. e-g, pY397FAK/tFAK levels for vinculin-null cells expressing vinculin constructs (top) and Western blots (below). e, 
For WT vinculin-expressing cells, pY397-FAK/tFAK levels (mean \pm SD) increase linearly with applied force. Linear regression $(P=0.0288, p Y 397-F A K / F A K=0.0801 *$ force +1.721$) . f, p Y 397-F A K /$ tFAK levels (mean \pm $\mathrm{SD})$ in vinculin-null cells are independent of external force. Linear regression $(P=0.4776)$. g, For cells expressing FAK E1015A, pY397-FAK/tFAK levels (mean \pm SD) are independent of external force. Linear regression $(P=0.5468)$.

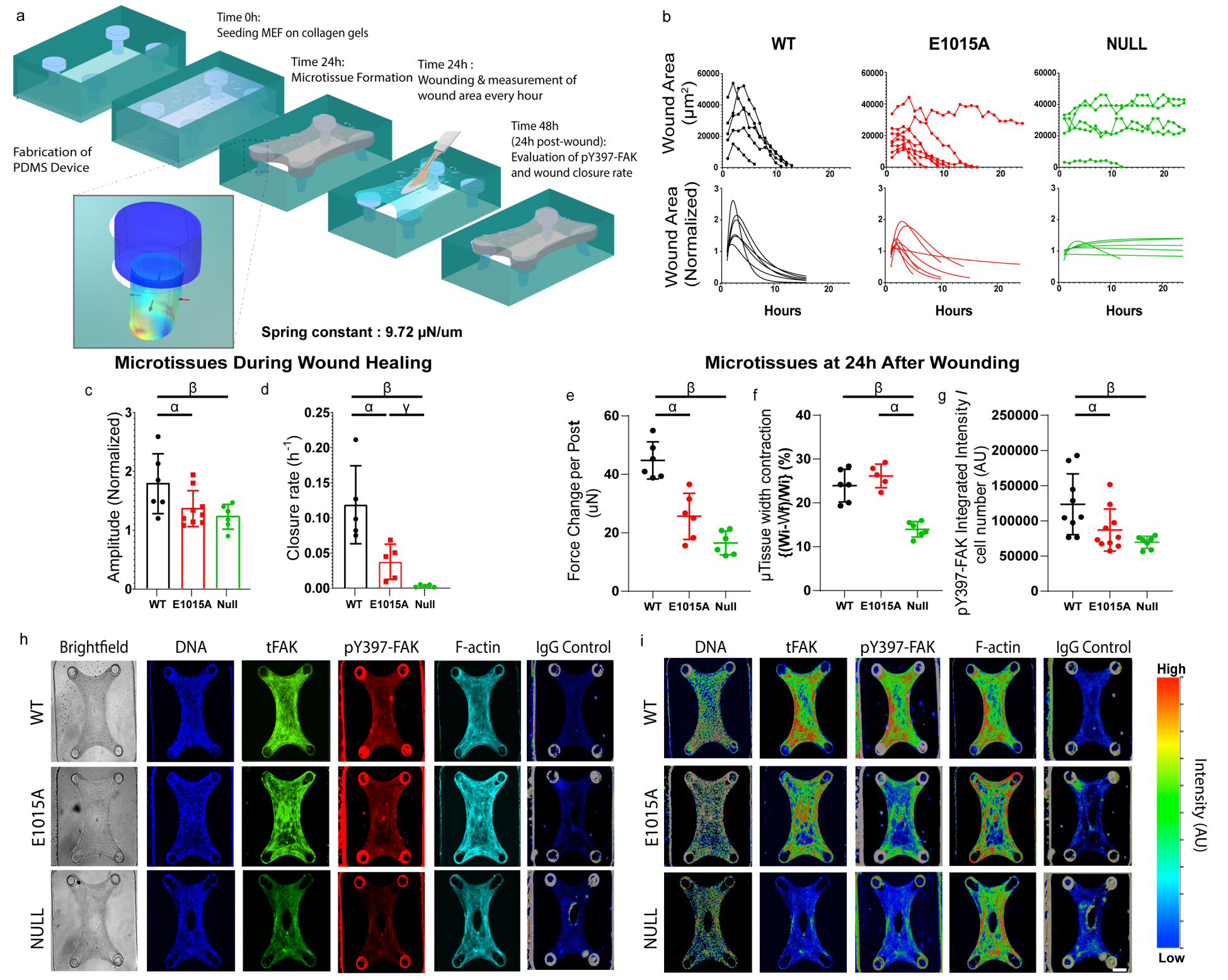

Figure 5

FAK-talin binding regulates wound closure of 3D microtissues. a, Schematic of microtissue assembly and wound closure assay. Collagen gels containing FAK-null cells expressing FAK constructs compact and form microtissue. At $24 \mathrm{~h}$ post-seeding, wound is created using microdissection knife, and wound closure is monitored over time. b, Wound area (top) and log-normal curve fit for wound area normalized to initial area (bottom) as a function of time for individual microtissues. c, Amplitude (mean \pm SD) of normalized wound area profile. Kruskal-Wallis test $P=0.0351, a: P=0.0440, \beta: P=0.0176$. $d$, Closure rate (mean $\pm S D$ ) of normalized wound area profile. ANOVA with Welch's correction $P=0.0064, a: P=0.0213, \beta$ : $P=0.0088, y$ : 
$\mathrm{P}=0.0289$. e, Net force difference (mean $\pm \mathrm{SD}$ ) between pre-wounding and $24 \mathrm{~h}$ post-wounding. KruskalWallis test $P<0.0001$, $a: P=0.0266, \beta$ : $P=0.0003$. $f$, Microtissue width contraction (mean $\pm S D$ ). KruskalWallis test $P=0.0002, a: P=0.0015, \beta: P=0.0101$. g, $p Y 397-F A K$ integrated intensity (mean $\pm S D$ ) at $24 \mathrm{~h}$ post-wounding. Kruskal-Wallis test $P=0.0056, \alpha: P=0.0232, \beta: P=0.0020$. $h$, Brightfield and fluorescence (DNA, tFAK, pY397-FAK, F-Actin, IgG control) images for microtissues at $24 \mathrm{~h}$ post-wounding. i, Intensity heatmaps (DNA, tFAK, pY397-FAK, F-Actin, IgG control) for microtissues. Scale bar $200 \mu \mathrm{m}$.

a
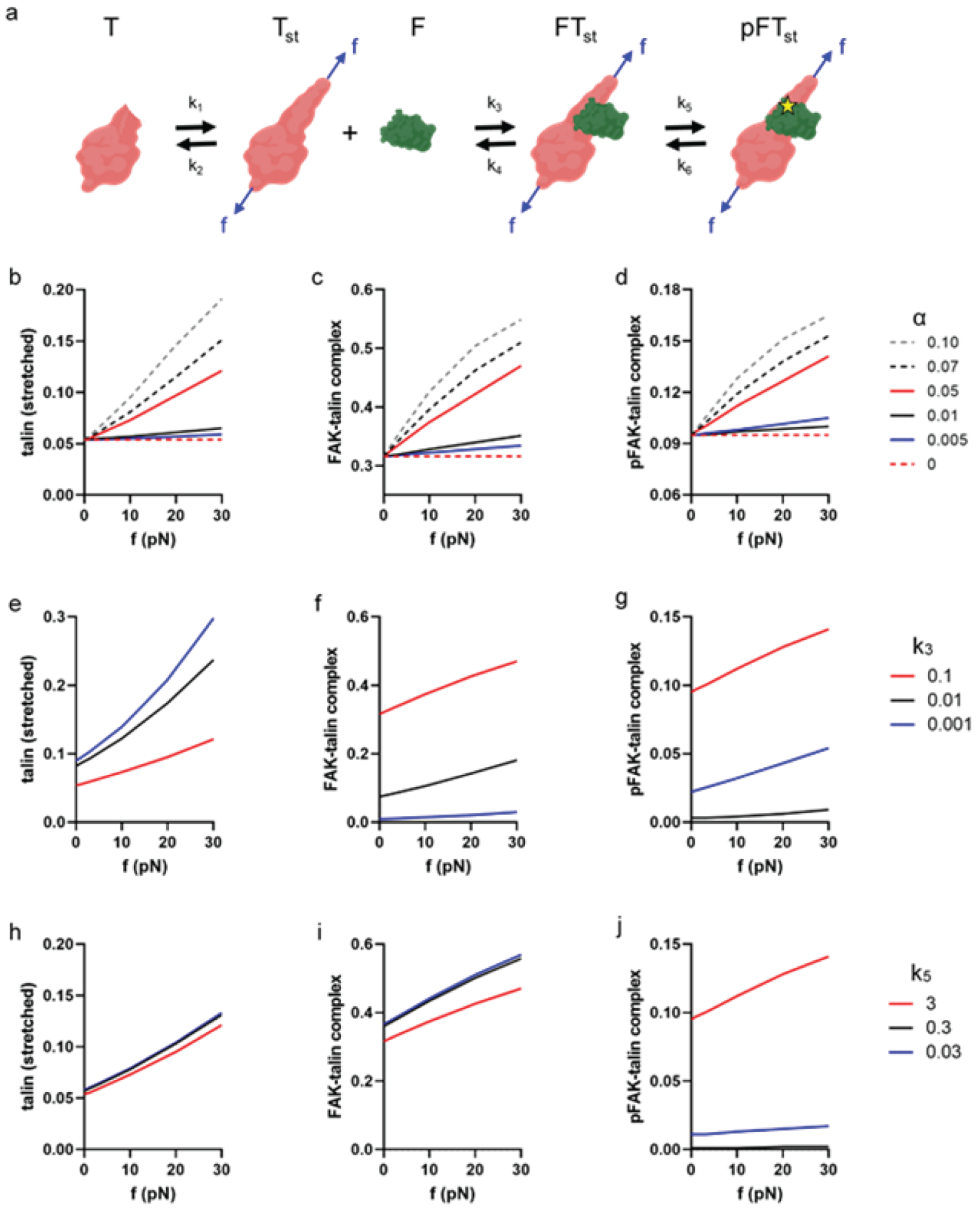

Figure 6 
Kinetic model of talin-FAK interactions under force. a, Schematic of FAK-talin interactions showing unstretched (T) and stretched talin (Tst), free FAK (F), FAK-talin complex (FTst), and phosphorylated FAKtalin complex (pFTst). Governing equations and parameters used in kinetic model are listed in Supplementary Materials. b-d, Solutions for relative number of (b) stretched talin, (c) FAK-talin complexes, and (d) phosphorylated FAK-talin complexes as a function of applied force ( $f$ ) for different values of the force coupling factor (a). e-g, Solutions for relative number of (e) stretched talin, (f) FAK-talin complexes, and $(\mathrm{g})$ phosphorylated FAK-talin complexes as a function of applied force ( $\mathrm{f}$ ) for different values of the forward binding rate constant for FAK-talin complex (k3). h-j, Solutions for relative number of $(h)$ stretched talin, (i) FAK-talin complexes, and (j) phosphorylated FAK-talin complexes as a function of applied force (f) for different values of the FAK phosphorylation rate constant (k5).

\section{Supplementary Files}

This is a list of supplementary files associated with this preprint. Click to download.

- FAKWTGFP.avi

- FAKE1015AGFP.avi

- FAKnullgFP.avi

- NMatRevisionSuppMaterials.pdf 